\title{
Fermi acceleration on the annular billiard: a simplified version
}

\author{
R Egydio de Carvalho, F Caetano de Souza and Edson D Leonel
}

Departamento de Estatística, Matemática Aplicada e Computação, Instituto de Geociências e Ciências Exatas, Universidade Estadual Paulista, UNESP Av.24A, 1515, Bela Vista 13506-700, Rio Claro, SP, Brazil

Received 8 November 2005, in final form 26 January 2006

Published 22 March 2006

Online at stacks.iop.org/JPhysA/39/3561

\begin{abstract}
A simplified version of a time-dependent annular billiard is studied. The dynamics is described using nonlinear maps and we consider two different configurations for the billiard, namely (i) concentric and (ii) eccentric cases. For the concentric case and for a null angular momentum, we confirm that the results for the Fermi-Ulam model are recovered and the particle does not experience the phenomenon of Fermi acceleration. However, on the eccentric case the particle demonstrates unlimited energy gain and Fermi acceleration is therefore observed.
\end{abstract}

PACS numbers: 05.45.-a, 05.45Pq, 05.45.Gg

\section{Introduction}

Dynamical systems described by nonlinear mappings have been studied and characterized by many authors during the past few years. An overview of a wide attention in this field can be found in [1-3]. A special area that has been extensively investigated on these systems is particularly related to the so-called conservative billiards problems. Generally, a billiard is defined by a connected region $Q \subset R^{D}$, with boundary $\partial Q \subset R^{D-1}$ which separates $Q$ from its complement. Inside the billiard, a point particle moves freely along a straight line until it hits a boundary. After the collision, it is also assumed that the particle is specularly reflected, in the sense that the incidence angle is equal to the reflection angle. Depending on the shape of the boundary, the dynamics of the particle might generate phase spaces of different kinds, including (i) regular, (ii) ergodic and (iii) mixed. The integrability of the regular cases, in general, appears due to the angular momentum preservation. On the other hand, in ergodic billiards, only chaotic and unstable periodic orbits are present in the dynamics and the so-called Bunimovich stadium $[4,5]$ consists of a typical example. In this system, the evolution of a single initial condition fills ergodically the entire phase space. Finally, there is a representative number of billiards that present the mixed phase space structure [6-9], which 
have control parameters with different physical significance. Depending on the combination of both initial conditions and control parameters, the phase space present a very rich structure which contains invariant spanning curves, Kolmogorov-Arnol'd-Moser (KAM) islands and chaotic seas.

There is a special allusion that might consider the boundary of a billiard as an infinite potential barrier. See, for example, [10] for recent results and scaling analysis for a classical particle in an infinite potential containing a time varying barrier. For static boundary, the particle's initial energy is always a constant. However, complex dynamics including energy and time may appear when a time periodic varying boundary is introduced. The main question to be answered is whether the system demonstrates unlimited energy gain, i.e. the basic condition for observing the phenomenon of Fermi acceleration [11]. Recently, Loskutov, Ryabov and Akinshin [12] studied specific billiards and on the basis of their results they have announced a conjecture (LRA-conjecture) that says a chaotic dynamics for a billiard with static boundary is a sufficient condition for the Fermi acceleration in the system when a time perturbation is introduced on the boundary. In connection with this conjecture, there has been a deep analysis of time-dependent stadium-like billiards [13] and we have studied a complete version of the annular billiard and, moreover, enlarged the number of cases for which such a conjecture has been applied with entire success [14]. Our main goal however in this paper is to introduce, for the first time, a simplified version of a time-dependent annular billiard. Historically, simplifications of moving boundaries in mappings are specially due to the seminal paper of Lieberman and Lichtenberg [15] where they develop a simplified version for the Fermi accelerator model. Since then, many other different models use basically the same approximation [16-23] and recently, Leonel and McClintock [24] proposed a hybrid version of both the Fermi-Ulam accelerator and bouncer models. The system behaves neither exclusively as pure Fermi-Ulam nor as a pure bouncer model, but as a combination of the two. They have used the simplified version of the model to obtain analytically the conditions for which properties that are individually present in the Fermi-Ulam and bouncer model but that come out and coalesce together in the hybrid version of the model.

This paper is organized as follows. In section 2, we present and discuss all the details needed for the construction of the mappings for the simplified version of the time-dependent annular billiard. In section 3, we present and discuss the numerical results while conclusion and final remarks are drawn in section 4.

\section{A simplified version of a time-dependent annular billiard}

It is well known that the annular billiard consists of a classical particle confined in an annular region limited by two circumscribed circles. The particle thus evolves in the ring between the two circles and suffers elastic collisions with the boundaries. We therefore introduce in this section a simplified version of the time-dependent annular billiard [14]. In a similar way as introduced by Lieberman and Lichtenberg [15], a simplified version of the model considers that both the internal and external boundaries are fixed, but that, after the particle collides with them, suffers a change in its velocity as if the boundaries were moving. With these simplifications, the instant of the impact, that in the complete version were obtained from solutions of transcendental equations, now are easily and directly obtained from, at most, second degree polynomial equations. Although this strategic simplification brings the huge advantage of allowing us speed up the numerical simulations, it also gives rise to a problem that we need necessarily to avoid. In the complete version, depending on the combination of both the velocity of the particle and phase of the moving boundary, it is possible for a particle, after suffering a collision with the boundary, suffers a second and successive collision 

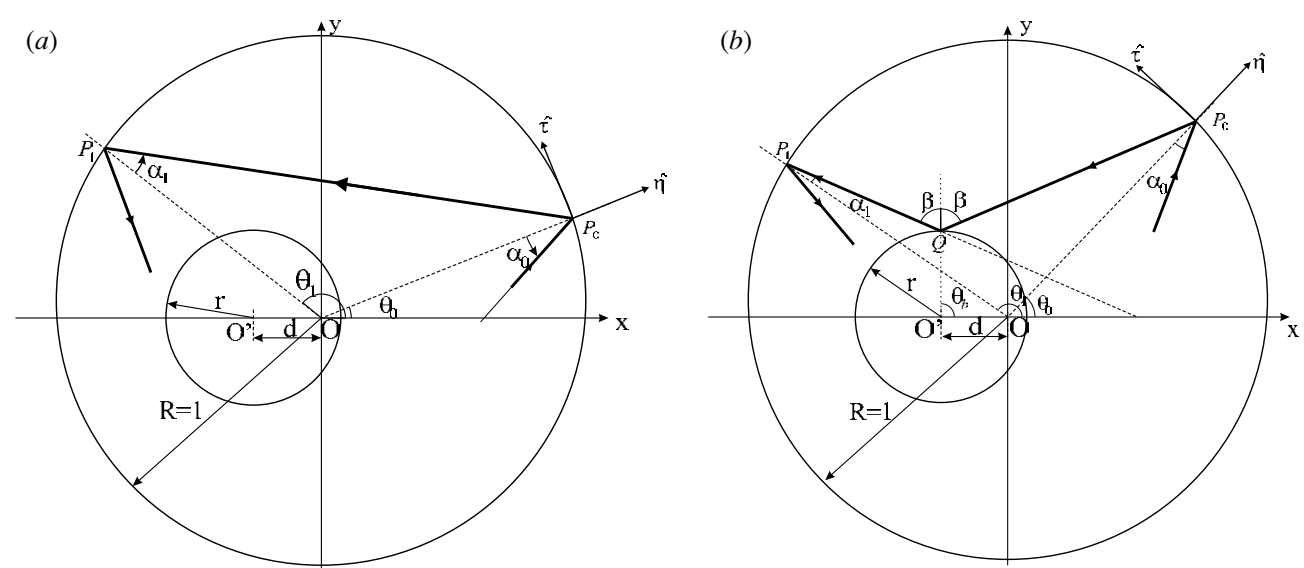

Figure 1. A geometric scheme of a particle in the model of the simplified time-dependent annular billiard: $(a)$ type A movement and $(b)$ type B movement.

before it leaves the collision area. It is also possible for a particle suffering further successive collisions and also having a positive (negative) radial velocity after the collision with the external (internal) boundary. In our simplified version however, successive collisions are forbidden because they would be interpreted as an equivalent to the particle moving beyond the boundaries. Therefore, after a collision with the external (internal) boundary, the particle has a positive (negative) radial velocity, we inject it back with same modulus of radial velocity.

Let us now construct the map. The derivation of the mapping follows, in part, the same general procedures as used in [12]. We thus define the radius of the outer circle as $R=1$, while for the inner circle, it is denoted by $r$. The eccentricity, defined as the distance between the centres of both circles, is represented by $d$. See figure 1 for a typical illustration of the annular billiard for the two possible types of movements. We also use the constraint $r+d<1$.

The dynamics is described using a nonlinear map $M\left(\theta_{n}, \alpha_{n}, v_{n}, \varphi_{n}, \phi_{n}\right)=$ $\left(\theta_{n+1}, \alpha_{n+1}, v_{n+1}, \varphi_{n+1}, \phi_{n+1}\right)$, where $n$ denotes the $n$th collision with the external boundary. The variable $\alpha_{n}$ is defined as the incidence angle and it is always measured with respect to the normal at the point of the impact. By convention, $\alpha_{n}$ is positive if measured counterclockwise and negative if clockwise. The corresponding range for $\alpha_{n} \in[-\pi / 2, \pi / 2]$. The variable $\theta_{n}$ gives the position of the particle on the boundary and it is considered, by convention, to be positive if measured counterclockwise and negative if measured clockwise. The range for $\theta_{n}$ is $\theta_{n} \in[-\pi, \pi]$. The variable $v_{n}$ denotes the velocity of the particle while both $\varphi_{n}$ and $\phi_{n}$ are the phases that will be used in the corresponding exchanges of momentum with the external and internal boundaries, respectively.

Supposing that after suffering a collision with the external boundary, the position of the particle, in rectangular coordinates are given by $x_{n}=\cos \left(\theta_{n}\right)$ and $y_{n}=\sin \left(\theta_{n}\right)$, the velocity, written in polar coordinates are $v_{n \eta}=-v_{n} \cos \left(\alpha_{n}\right)$ and $v_{n \tau}=v_{n} \sin \left(\alpha_{n}\right)$, where the index $\eta$ represents the normal component of the velocity (the unit vector is defined as pointing outside) and $\tau$ denotes the tangential component of the velocity. Moreover, in rectangular Cartesian coordinates, the velocity is given by

$$
v_{n x}=v_{n \eta} \cos \left(\theta_{n}\right)-v_{n \tau} \sin \left(\theta_{n}\right), \quad v_{n y}=v_{n \eta} \sin \left(\theta_{n}\right)+v_{n \tau} \cos \left(\theta_{n}\right) .
$$

The two possible kinds of movements (see figure 1) are obtained from the tangency condition $[25,26]$

$$
\left|\sin \left(\alpha_{n}\right)-d \sin \left(\theta_{n}-\alpha_{n}\right)\right|>r
$$


If the combination of both $\theta_{n}$ and $\alpha_{n}$ are such that condition (1) is satisfied, then we have a movement of type A, otherwise the movement is of type B. Let us first discuss the type A.

Type A The particle does not hit the internal boundary.

If the particle does not collides with the internal circle, then the next collision will be with the external boundary. The position of the particle in the external circle, in rectangular Cartesian coordinates, is given by $x_{n+1}=\cos \left(\theta_{n+1}\right)$ and $y_{n+1}=\sin \left(\theta_{n+1}\right)$ with

$$
\theta_{n+1}=\theta_{n}+\pi-2 \alpha_{n} .
$$

We can define the flight time between the two collisions evaluating the equation

$$
t_{f}=\frac{\Delta S}{v_{n}}=\frac{\sqrt{\left(x_{n+1}-x_{n}\right)^{2}+\left(y_{n+1}-y_{n}\right)^{2}}}{v_{n}} .
$$

Immediately before the collision with the external boundary and considering polar coordinates, the components for the velocity of the particle are written as

$v_{n \eta}=v_{n x} \cos \left(\theta_{n+1}\right)+v_{n y} \sin \left(\theta_{n+1}\right), \quad v_{n \tau}=-v_{n y} \sin \left(\theta_{n+1}\right)+v_{n y} \cos \left(\theta_{n+1}\right)$.

After the impact and according to our definition of the simplified version, the new component of the radial velocity is given by

$$
v_{(n+1) \eta}=-\left|-v_{n \eta}-2 \epsilon_{R} \sin \left(t_{f}+\varphi_{n}\right)\right|, \quad v_{(n+1) \tau}=v_{n \tau} .
$$

In the complete version, $\epsilon_{R}$ represents the amplitude of oscillation of the external moving boundary. By definition of the simplified version, we have assumed that both the boundaries are fixed but the particle suffers an exchanges of momentum after the collision as if the boundaries were moving. Then the expression for the velocity of the particle is the same as the one used in the complete version, except for the module function, which is particular of the simplified model. We emphasize however that the module is an artificial strategy used to avoid the particle moving beyond the boundary. After the collision, the new velocity is then represented as $v_{n+1}=\sqrt{v_{(n+1) \eta}^{2}+v_{(n+1) \tau}^{2}}$. Finally, the corresponding phases and reflection angle are

$$
\begin{aligned}
& \varphi_{n+1}=\varphi_{n}+t_{f}, \quad \bmod 2 \pi \\
& \phi_{n+1}=\phi_{n}+\omega t_{f}, \quad \bmod 2 \pi \\
& \alpha_{n+1}=\arctan \left[-\frac{v_{(n+1) \tau}}{v_{(n+1) \eta}}\right] .
\end{aligned}
$$

We now discuss the situation where condition (1) is not matched.

Type B In this case, after the particle collides with the external boundary, it hits necessarily the internal circle.

To avoid confusion, we emphasize that the index ' $n$ ' counts the hits with the outer circle. Even then, we keep it in the variables when the particle hits the inner circle too. However, the iterations of the map are computed only when the external boundary is reached. The rectangular Cartesian coordinates of the particle in the instant of the impact with the internal circle are given by

$$
x_{r}=r \cos \left(\theta_{b}\right)-d, \quad y_{r}=r \sin \left(\theta_{b}\right),
$$

with $\theta_{b}=\beta+\theta_{n}-\alpha_{n}$ and $\beta$ obtained from

$$
\sin (\beta)=\frac{1}{r}\left[\sin \left(\alpha_{n}\right)-d \sin \left(\theta_{n}-\alpha_{n}\right)\right] .
$$


The flight time, up to the internal boundary, is then

$$
t_{f}=\frac{\Delta S}{v_{n}}=\frac{\sqrt{\left(x_{r}-x_{n}\right)^{2}+\left(y_{r}-y_{n}\right)^{2}}}{v_{n}},
$$

where $v_{n}$ is the velocity of the particle before the collision, and the corresponding polar components of the velocity are given by

$$
v_{n \eta}^{r}=v_{n x} \cos \left(\theta_{b}\right)+v_{n y} \sin \left(\theta_{b}\right), \quad v_{n \tau}^{r}=-v_{n x} \sin \left(\theta_{b}\right)+v_{n y} \cos \left(\theta_{b}\right) .
$$

In the referential frame of the internal circle, only the radial component of the velocity changes. In this way, immediately after the collision with the internal boundary, the corresponding expressions are

$$
v_{(n+1) \eta}^{r}=\left|-v_{n \eta}^{r}-2 \epsilon_{r} \omega \sin \left(\omega t_{f}+\phi_{n}\right)\right|, \quad v_{(n+1) \tau}^{r}=v_{n \tau}^{r} .
$$

The upper index $r$ indicates that the position of the particle is measured on the internal circle. A similar discussion holds here for the variable $\epsilon_{r}$. On the complete version, it denotes the amplitude of oscillation of the internal time varying boundary. Therefore, it is assumed that, after the impact, the expression of the particle's velocity is the same as that used in the complete model. Again, we stress that the module function is introduced to avoid the particle moving into forbidden regions. The frequency $\omega$ in fact represents the radio of the internal and external oscillating frequencies. See [14] for a full discussion. After the collision, the particle goes straight to the external boundary located at $R=1$. The collision then happens if the position of the particle is equal to the position of the external circle, i.e.

$$
R_{p}(t)=1 \text {, }
$$

where $R_{p}(t)$ denotes the module vector radius of the particle, which is given by $R_{p}(t)=$ $\sqrt{\left(x_{r}^{2}+y_{r}^{2}\right)+2\left(x_{r} v_{x}+y_{r} v_{y}\right) t+\left(v_{x}^{2}+v_{y}^{2}\right) t^{2}}$ where the velocities are obtained from

$$
\begin{aligned}
& v_{x}=v_{(n+1) \eta}^{r} \cos \left(\theta_{b}\right)-v_{(n+1) \tau}^{r} \sin \left(\theta_{b}\right), \\
& v_{y}=v_{(n+1) \eta}^{r} \sin \left(\theta_{b}\right)+v_{(n+1) \tau}^{r} \cos \left(\theta_{b}\right) .
\end{aligned}
$$

With these definitions, condition (4) might be written as

$$
\left(v_{x}^{2}+v_{y}^{2}\right) t^{2}+2\left(x_{r} v_{x}+y_{r} v_{y}\right) t+\left(x_{r}^{2}+y_{r}^{2}\right)-1=0 .
$$

The largest solution of the above equation, we call it as $t_{c}$, gives the time that the particle spends after a collision with the internal circle until it reaches the external boundary. The corresponding new rectangular Cartesian coordinates for the particle are

$$
x_{n+1}=x_{r}+v_{x} t_{c}, \quad y_{n+1}=y_{r}+v_{y} t_{c} .
$$

The new angle $\theta_{n+1}$ is obtained from the equation

$$
\theta_{n+1}=\arctan \left[\frac{y_{n+1}}{x_{n+1}}\right]
$$

and the corresponding components of the particle's velocity immediately before the impact with the outer circle are

$$
\begin{aligned}
& v_{n \eta}=v_{x} \cos \left(\theta_{n+1}\right)+v_{y} \sin \left(\theta_{n+1}\right), \\
& v_{n \tau}=-v_{x} \sin \left(\theta_{n+1}\right)+v_{y} \cos \left(\theta_{n+1}\right) .
\end{aligned}
$$

After the collision, only the radial component of the velocity is changed and as we have discussed for the simplified version, the expressions are

$$
v_{(n+1) \eta}=-\left|-v_{n \eta}-2 \epsilon_{R} \sin \left(t_{c}+t_{f}+\varphi_{n}\right)\right|, \quad v_{(n+1) \tau}=v_{n \tau} .
$$


The module function is introduced to maintain the position of the particle inside the external boundary. Finally, the corresponding new velocity is written as $v_{n+1}=\sqrt{v_{(n+1) \eta}^{2}+v_{(n+1) \tau}^{2}}$. The corresponding phases are

$$
\begin{array}{ll}
\varphi_{n+1}=\varphi_{n}+t_{c}+t_{f}, & \bmod 2 \pi \\
\phi_{n+1}=\phi_{n}+\omega\left(t_{c}+t_{f}\right), & \bmod 2 \pi .
\end{array}
$$

On the basis of the expressions of the mappings, we are now able to present and discuss the numerical results.

\section{Numerical results}

We discuss in this section the results obtained for our simplified version of the annular billiard. First, the concentric case is considered and second the results for the eccentric case are presented and discussed.

\subsection{Concentric case}

On the concentric case, the control parameter $d$ is fixed as $d=0$. As a similar discussion for the complete version (see [14]), the initial condition $\alpha_{n}$ is obtained from the equation

$$
\sin \left(\alpha_{n}\right)=\frac{l}{\left(1+\epsilon_{R} \cos \left(\varphi_{n}\right)\right) v_{n}},
$$

where there is a special range of values for the angular momentum $l$. For large enough values of $l$, the particle suffers collision with the internal circle only for the regime of high energy. We therefore set the range of $l$ as $l \in\left[0, \epsilon_{R}\right]$. For $l=0$, our model recovers all the results of the Fermi-Ulam accelerator model [1]. The upper limit $\epsilon_{R}$ denotes the maximum value of the velocity for the corresponding complete version of the model with external moving boundary.

To illustrate the validity of the model, we show in figure 2 the corresponding phase space, in the variables $\left(-v_{\eta}, \varphi\right)$, for different configurations of control parameters and initial conditions. It is easy however to see in figure 2 that the mixed structure of the phase space is present. For the regime of low energy, the iteration of a single initial condition is enough to fill the chaotic area. In addition, KAM islands are present and, sometimes, they are also surrounded by the chaotic sea. Moreover, the phenomenon of Fermi acceleration is not observed mainly due to the presence of a set of invariant spanning curves which limit the size of the chaotic sea.

The presence of invariant spanning curves necessarily implies that the chaotic sea presents a typical value for the average velocity. It might be averaged both as a function of an ensemble of different initial conditions and over the orbit. Consequently, starting from very low values of the velocity and averaging it over the orbit, we can see that (see figure 3) the velocity grows for low iteration number and then after a changeover, the curve of the average velocity bends towards a regime of saturation. Such a regime is only reached for long enough iterations. The behaviour of the average velocity as function of the iteration number for different configurations is shown in figure 3. The control parameters used are $r=0.5$, $\epsilon_{R}=\{0.01,0.001\}$ and (a) $\epsilon_{r}=\epsilon_{R}, \omega=0.5, \varphi_{0}-\phi_{0}=0$; (b) $\epsilon_{R}=\epsilon_{r}, \omega=2, \varphi_{0}-\phi_{0}=0$; (c) $\epsilon_{R}=\epsilon_{r}(\sqrt{5}+1) / 2, \omega=(\sqrt{5}+1) / 2$ and $\varphi_{0}-\phi_{0}=(\sqrt{5}+1) / 2$.

We have also studied the behaviour of the average velocity and maximum value for the velocity on the chaotic sea as function of $\omega$. The angular frequency however appears explicitly on the expression of the velocity of the particle after it suffers a collision with the internal boundary. Therefore, an increase in $\omega$ also implies an increase in the maximum value for the 
(a)

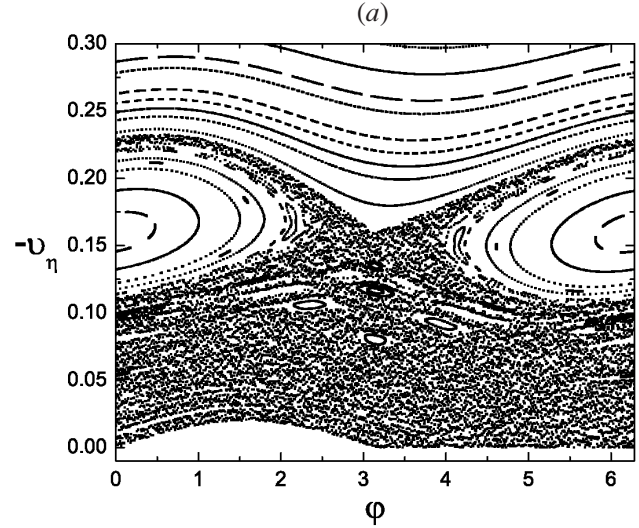

(c)

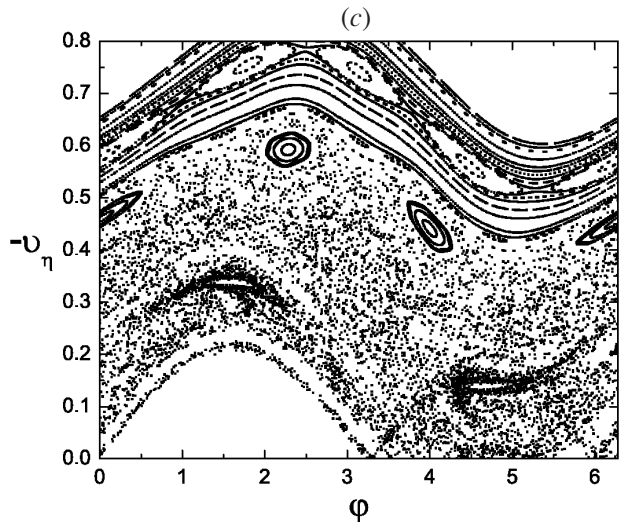

(b)

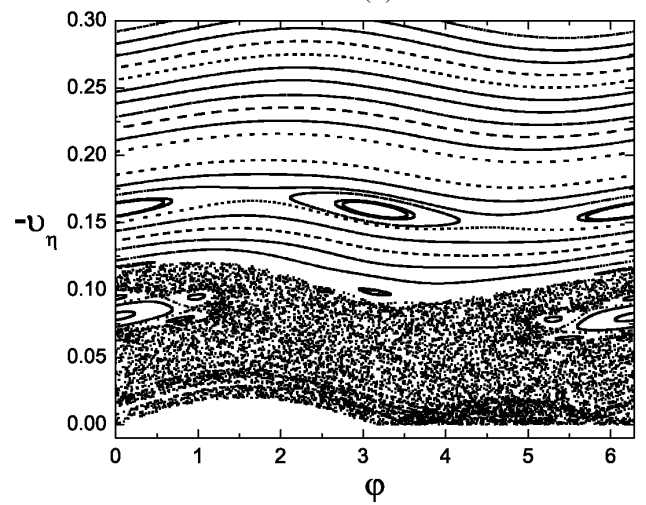

(d)

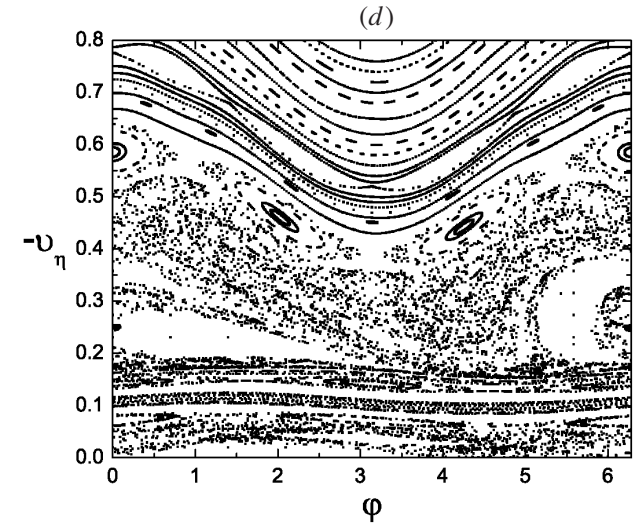

Figure 2. Phase space for the simplified version of the time-dependent, concentric $(d=0)$ annular billiard on the variables $\left(-v_{\eta}, \varphi\right)$. The control parameters used were $r=0.5, \omega=1$ and: (a) $\epsilon_{R}=\epsilon_{r}=0.01, l=0.01$ and $\varphi_{0}-\phi_{0}=0 ;(b) \epsilon_{R}=\epsilon_{r}=0.01, l=0.01$ and $\varphi_{0}-\phi_{0}=\pi$; (c) $\epsilon_{R}=0.1, \epsilon_{r}=0.01, l=0.1$ and $\varphi_{0}-\phi_{0}=0 ;(d) \epsilon_{R}=0.01, \epsilon_{r}=0.1, l=0.1$ and $\varphi_{0}-\phi_{0}=0$.

velocity in a chaotic orbit for the regime of low energy. Since an increase in $\omega$ implies an increase of the exchange of momentum (see equation (3)), it is expected that the position of the lowest invariant spanning curve will also rise. The behaviour of both the maximum and medium velocities as function of $\omega$ is shown in figure 4 . The medium velocity was obtained as follows: we iterate an ensemble of $10^{3}$ different initial conditions along $10^{6}$ iterations. Then, the curve approaches the saturation; we have obtained the saturation value for different values of $\omega$ used. The maximum value of the velocity however is collected numerically for a long run with an initial condition in the chaotic low energy region.

Let us now discuss the origin of the linear behaviour for the maximum velocity as function of the angular frequency $\omega$ (see figure 4 ). We will consider that our model, in the neighbouring of the lowest invariant spanning curve (around $-v_{\eta} \cong 0.23$ for figure $2(a)$ ), is locally described via the standard map [1]

$$
I_{n+1}=I_{n}+K \sin \left(\Theta_{n}\right), \quad \Theta_{n+1}=\Theta_{n}+I_{n+1}, \quad \bmod 2 \pi .
$$

It is well known that the standard model presents a transition for $K \approx 0.97 \ldots$ For $K \leqslant 0.97 \ldots$ the phase space presents invariant spanning curves which limit the size of 
(a)

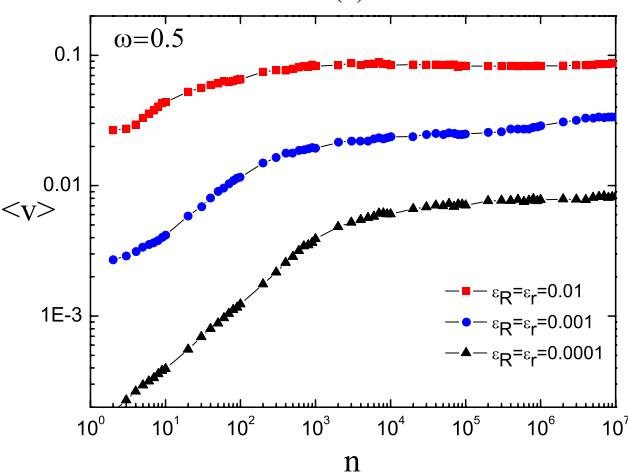

(c)

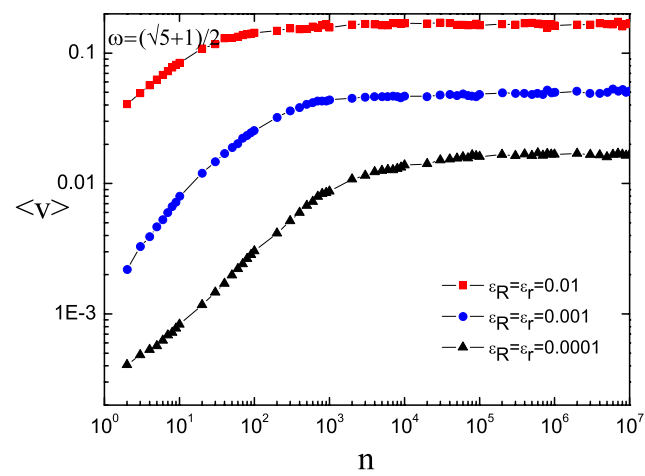

(b)

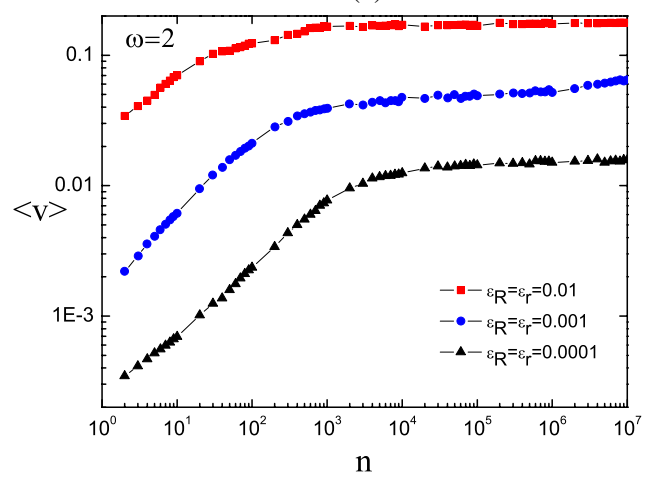

$\mathrm{n}$

Figure 3. Behaviour of the average velocity as function of the iteration number for the concentric case. The control parameters used are $r=0.5$ and $\epsilon_{R}=\{0.01,0.001\}$ and (a) $\epsilon_{r}=\epsilon_{R}, \omega=0.5, \varphi_{0}-\phi_{0}=0 ;(b) \epsilon_{R}=\epsilon_{r}, \omega=2$ and $\varphi_{0}-\phi_{0}=0 ;(c) \epsilon_{r}=\epsilon_{R}(\sqrt{5}+1) / 2$, $\omega=(\sqrt{5}+1) / 2$ and $\varphi_{0}-\phi_{0}=(\sqrt{5}+1) / 2$.

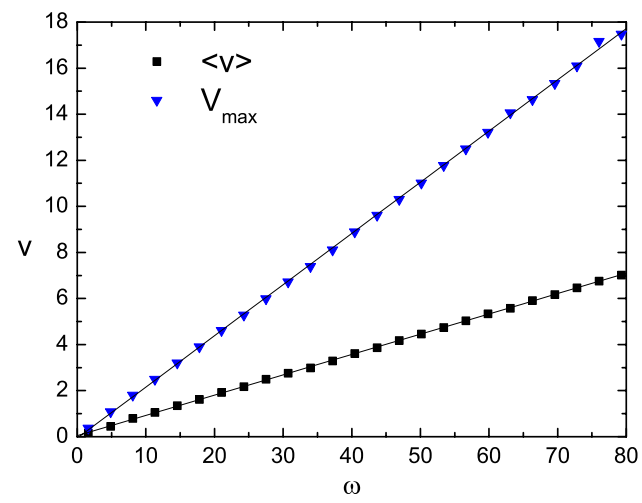

Figure 4. Velocity as function of $\omega$. The control parameters used were $r=0.5, \epsilon_{r}=0.01, \epsilon_{R}=$ $\epsilon_{r}(\sqrt{5}+1) / 2, \varphi_{0}-\phi_{0}=(\sqrt{5}+1) / 2$.

the chaotic sea. Therefore, for $K>0.97 \ldots$, the invariant spanning curves are completely destroyed. Our approach consists in determining, for the present version of the model, an effective constant $K_{\text {eff }}$ which approximately locates the position of the lowest invariant 
spanning curve. We will consider, without loss of generality, the expression of the velocity immediately after the collision with the internal boundary, $v_{n+1}$, which is written as

$$
v_{n+1}^{2}=v_{(n+1) \eta}^{2}+v_{(n+1) \tau}^{2} .
$$

The main reason for using the internal boundary is that the angular frequency $\omega$ appears explicitly on the expression of exchange of velocity. Using equation (3), the above expression is rewritten according to

$$
v_{n+1}^{2}=\left(-v_{n \eta}-2 \epsilon_{r} \omega \sin \left(\phi_{n+1}\right)\right)^{2}+v_{n \tau}^{2} .
$$

For simplicity, we have omitted the upper index ' $r$ '. In a more appropriate form, equation (5) is

$$
v_{n+1}^{2}=v_{n}^{2}+4 \epsilon_{r} \omega \sin \left(\phi_{n+1}\right)\left[v_{n \eta}+\epsilon_{r} \omega \sin \left(\phi_{n+1}\right)\right],
$$

where $v_{n}^{2}=v_{n \eta}^{2}+v_{n \tau}^{2}$. We suppose that, in the invariant spanning curve, the velocity is given as $v_{n}=v^{*}+\Delta v_{n}$, where $v^{*}$ represents a typical value in the spanning curve and $\Delta v_{n}$ denotes its corresponding range of oscillation. On the basis of this supposition the phase $\phi_{n+1}$ is written, using equation (2), as

$$
\phi_{n+1}=\phi_{n}+\omega \frac{\Delta d}{v^{*}}\left(1+\frac{\Delta v_{n}}{v^{*}}\right)^{-1} .
$$

It is worth mentioning that the corresponding range of possible values for $\Delta d$ is

$$
\Delta d \in\left[\left(1-\epsilon_{R}\right)-\left(r+\epsilon_{r}\right),\left(1+\epsilon_{R}\right)-\left(r-\epsilon_{r}\right)\right] .
$$

Expanding equation (7) and taking only terms of first order in $\Delta v_{n} / v^{*}$, we obtain

$$
\phi_{n+1}=\phi_{n}+\omega \frac{\Delta d}{v^{*}}-\omega \Delta d \frac{\Delta v_{n}}{v^{* 2}} .
$$

Equation (8) allows us now to define the variable $I_{n}$ as

$$
I_{n}=\omega \frac{\Delta d}{v^{*}}-\omega \Delta d \frac{\Delta v_{n}}{v^{* 2}} .
$$

Let us then use the expression $v_{n}=v^{*}+\Delta v_{n}$ on equation (6). It is moreover written as

$$
\left(v^{*}+\Delta v_{n+1}\right)^{2}=\left(v^{*}+\Delta v_{n}\right)^{2}+4 \epsilon_{r} \omega \sin \left(\phi_{n+1}\right)\left[v_{n \eta}+\epsilon_{r} \omega \sin \left(\phi_{n+1}\right)\right] .
$$

Expanding both the squares and taking only term of first order for $\Delta v_{n} / v^{*}$, equation (9) becomes

$$
\Delta v_{n+1}=\Delta v_{n}+\frac{2 \epsilon_{r} \omega}{v^{*}} \sin \left(\phi_{n+1}\right)\left[v_{n \eta}+\epsilon_{r} \omega \sin \left(\phi_{n+1}\right)\right] .
$$

Multiplying both sides by $-\omega \Delta d / v^{* 2}$ and adding $\omega \Delta d / v^{*}$, respectively, equation (10) becomes

$$
I_{n+1}=I_{n}-\frac{2 \epsilon_{r} \omega^{2} \Delta d}{v^{* 3}} \sin \left(\phi_{n+1}\right)\left[v_{n \eta}+\epsilon_{r} \omega \sin \left(\phi_{n+1}\right)\right] .
$$

Let us now discuss the limits for the term inside the brackets in equation (11). Near the lowest invariant spanning curve we will suppose that the term $v_{n \eta}+\epsilon_{r} \omega \sin \left(\phi_{n+1}\right) \cong v^{*}$. Such a supposition is entirely confirmed in figure 5. It is shown in figure 5(a) the behaviour of the total velocity $\left(v_{n} \times n\right)$ while figure $5(b)$ presents the results of $\left(\left[v_{n \eta}+\epsilon_{r} \omega \sin \left(\phi_{n+1}\right)\right] \times n\right)$. It is important to stress however that the approximation via the standard map is valid only for values of velocities close to those shown in the invariant spanning curve (around $v \approx 0.23$ in figure 6). In this limit, $v_{n} \cong v^{*} \cong v_{n \eta}+\epsilon_{r} \omega \sin \left(\phi_{n+1}\right)$. According to the above discussion, we 


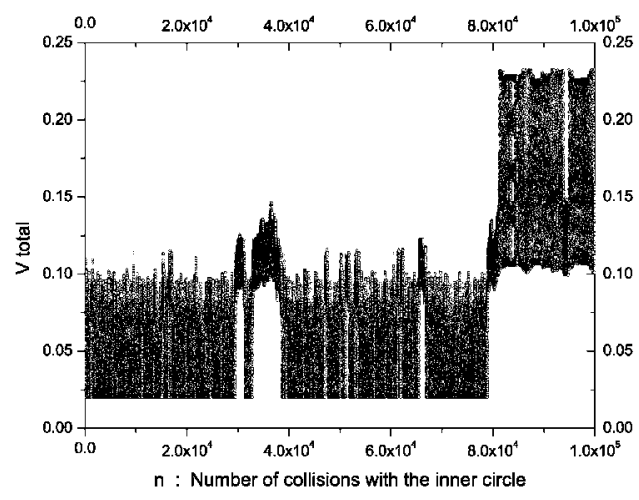

(a)

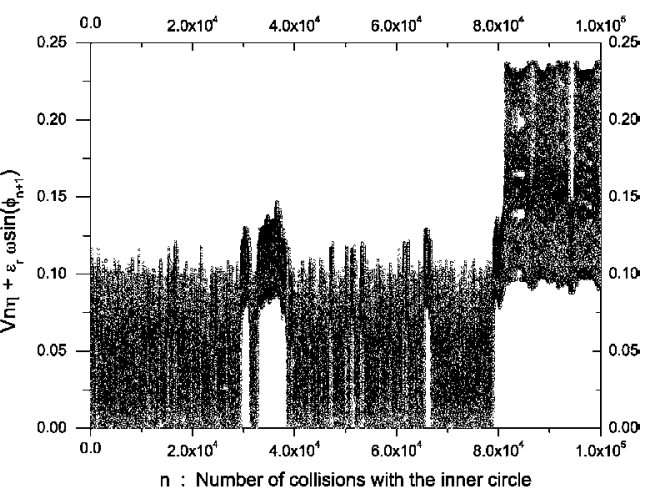

(b)

Figure 5. (a) Total velocity as a function of the collision number on the internal boundary. (b) The term in the brackets of equation (11) as function of the collision number on the internal boundary. The control parameters used in both figures were $r=0.5, d=0, \omega=1, \epsilon_{R}=\epsilon_{r}=0.01$ and $l=0.01$

can define a new $\Theta_{n}=\phi_{n+1}+\pi$, and obtain an effective constant $K_{\text {eff }}$ for our description of the problem as

$$
K_{\mathrm{eff}}=\frac{2 \epsilon_{r} \omega^{2} \Delta d}{v^{* 2}}
$$

Hence, the equations of the standard map are recovered with $K_{\text {eff }}$ in the place of $K$.

Moreover, around the transition on the standard map, the constant $K$ assumes the value $K \approx 0.97 \ldots$. Considering such a value for our results and assuming that $v_{\max }$ can now be approximate to $v^{*}$ (this approximation is valid only if $\Delta v_{n}$ is small enough), the expression for the maximum velocity is then given by

$$
v_{\max }=\sqrt{\frac{2 \epsilon_{r} \Delta d}{0.97 \ldots}} \omega .
$$

Therefore, our main conclusion of this discussion is that $v_{\max } \propto \omega$, which is an analytical explanation for the behaviour observed in figure 4.

We stress that the mixed phase space structure can be seen in the variables $\left(v_{n} \times \varphi_{n}\right)$, as is shown in figure 6 , where $v_{n}$ is the total velocity before the collision with the inner circle. The corresponding control parameters used in the construction of this figure were $r=0.5, d=0, \omega=1, \epsilon_{R}=\epsilon_{r}=0.01$ and $l=0.01$.

There are two main conclusions that we can extract from the results discussed in this section. The first of them is that, even considering a simplified version, our results reinforce the LRA-conjecture [12]. For the static version of the concentric case, it is well known that the system is integrable. The introduction of the time dependence on the exchange of velocity however does not imply that the particle will present unlimited energy gain. Such results are clearly shown in figures 3 and 4. The second conclusion refers to the connection of the standard map with our simplified version of the time-dependent annular billiard in order to describe locally the dynamics of the chaotic sea close the lowest invariant spanning curve. We have used our simplified version for explaining analytically the behaviour of the maximum value for the velocity in the chaotic sea for low energy domain. We will now discuss the eccentric case. 


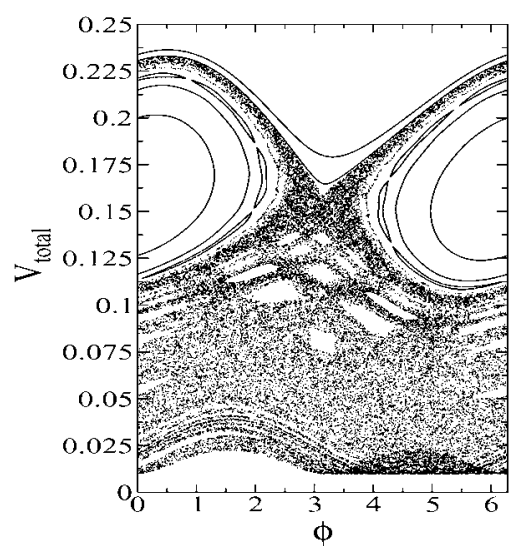

Figure 6. Phase space on the variables $v_{n} \times \varphi_{n}$. The mixed structure is also present and it is easy to compare the maximum value of the velocity on the chaotic sea as those shown in figures $5(a)$ and $(b)$. The control parameters used were $r=0.5, d=0, \omega=1, \epsilon_{R}=\epsilon_{r}=0.01$ and $l=0.01$.

(a)

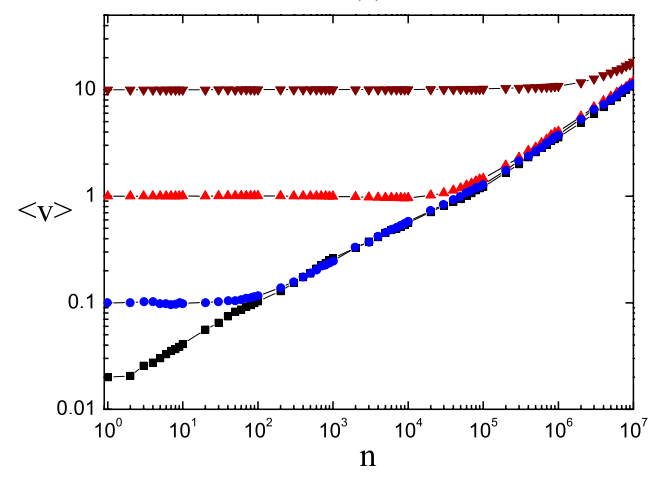

(b)

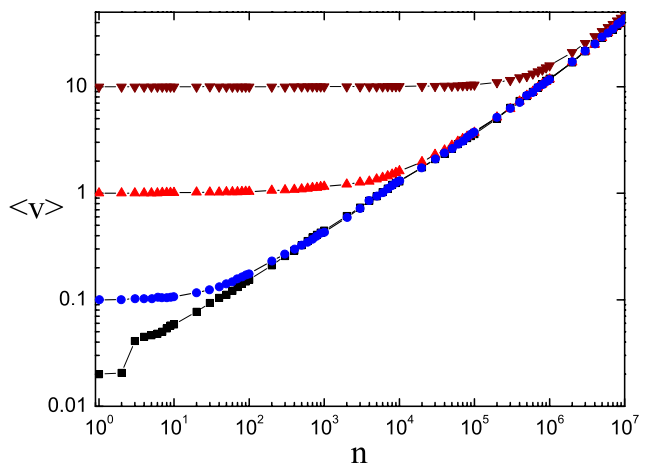

Figure 7. Behaviour of the average velocity as function of the iteration number for the eccentric case. The control parameters used were $r=0.05, d=0.65, \epsilon_{R}=0.01, v_{0}=$ $\left\{2 \epsilon_{r}, 10 \epsilon_{R}, 100 \epsilon_{R}, 1000 \epsilon_{R}\right\}$ and $(a) \omega=1, \epsilon_{r}=\epsilon_{R} ;(b) \epsilon_{r}=\epsilon_{R}(\sqrt{5}+1) / 2$ and $\omega=(\sqrt{5}+1) / 2$.

\subsection{Eccentric case}

Let us now discuss our numerical results obtained for the eccentric case. For easier comparisons with the results of the complete version of the model we will consider only two sets of control parameters as those used in [14], as can be seen in figure 7. The behaviour of the average velocity as function of the iteration number is shown in figure 7 . We have evolved up to $10^{7}$ iterations, an ensemble of $10^{3}$ different initial conditions. The initial velocities are shown in figure 7 but the phases were uniformly distributed between $\varphi_{0}$ and $\phi_{0} \in[0,2 \pi]$.

The main conclusion for this section is that, even using our simplified model, the system presents the phenomenon of Fermi acceleration. We stress that for the static version of the model, the system has a mixed structure for the phase space. The introduction of time dependency does imply that the phenomenon of Fermi acceleration is then observed, according to the LRA-conjecture [12]. 


\section{Final remarks and conclusions}

We have proposed and studied for the first time, a simplified version of the time-dependent annular billiard under two different configurations: (i) concentric and (ii) eccentric cases. In the static case it is well known that the annular billiard is integrable if the circles are concentric and it presents mixed phase space structure if they are eccentric. The system is described by nonlinear mappings and we have shown that for the time-dependent concentric case, the phase space is of mixed kind for the variables $\left(v_{\eta}, \varphi\right)$ and also for $\left(v_{\text {total }}, \varphi\right)$. Therefore, the Fermi acceleration does not occur. In addition, we have derived an analytical expression that describes the behaviour of the maximum velocity as function of the angular frequency $\omega$ at energies (velocities) near the energy of the lowest spanning curve $\left(-v_{\eta} \approx 0.23\right.$ in figures $2(a)$ and 6). Consequently, in this neighbourhood the system is well represented by a standard maplike. On the other hand, for the eccentric case the Fermi acceleration is observed, which reinforces the LRA-conjecture [12], and it consists in our main conclusion in this paper. The introduction of a simplified version of the time-dependent annular billiard is also in total agreement with the exact results obtained from the complete version of this billiard (see [14] for a full discussion).

Finally, we also would like to point out that for very small eccentricities (the static case is non-integrable) the Fermi acceleration was not observed, for the set of control parameters we have used. Moreover, we evaluate that if the time-dependent perturbation was sufficiently increased the Fermi acceleration might be observed. This question will be considered in a future paper.

\section{Acknowledgments}

This research was partially supported by the scientific Brazilian agencies, Conselho Nacional de Desenvolvimento Científico e Tecnológico-CNPq and Coordenação de Aperfeiçoamento de Pessoal de Nível Superior-CAPES.

\section{References}

[1] Lichtenberg A J and Lieberman M A 1992 Regular and Chaotic Dynamics (Applied Mathematical Science vol 38) (New York: Springer)

[2] Guckenheimer J and Holmes P 2000 Nonlinear Oscillations, Dynamical Systems, and Bifurcations of Vector Fields (Appl. Math. Sci. vol 42) (New York: Springer)

[3] Bai-Lin H 1990 Chaos II (Singapore: World Scientific)

[4] Bunimovich L A 1974 Math. USSR Sb. 2345

[5] Bunimovich L A 1979 Commun. Math. Phys. 65295

[6] Berry M V 1981 Eur. J. Phys. 291

[7] Kamphorst S O and Carvalho S P 1999 Nonlinearity 121363

[8] Robnik M 1983 J. Phys. A: Math. Gen. 163971

[9] Robnik M and Berry M V 1985 J. Phys. A: Math. Gen. 181361

[10] Leonel E D and McClintock P V E 2005 Chaos 15033701

[11] Fermi E 1949 Phys. Rev. 751169

[12] Loskutov A, Ryabov A B and Akinshin L G 2000 J. Phys. A: Math. Gen. 337973

[13] Loskutov A and Ryabov A 2002 J. Stat. Phys. 108995

[14] Egydio de Carvalho R, Caetano de Souza F and Leonel E D 2006 Fermi acceleration on the annular billiard to appear

[15] Lieberman M A and Lichtenberg A L 1972 Phys. Rev. A 51852

[16] Holmes P J 1982 J. Sound Vib. 84173

[17] Tsang K Y and Lieberman M A 1984 Physica D 11147

[18] Everson R M 1986 Physica D 19355 
[19] Lieberman M A and Tsang K Y 1985 Phys. Rev. Lett. 55908

[20] Tsang K Y and Lieberman M A 1986 Physica D 21401

[21] Tsang K Y and Ngai K L 1997 Phys. Rev. E 56 R17

[22] Leonel E D, da Silva J K L and Kamphorst S O 2004 Physica A 331435

[23] Leonel E D, McClintock P V E and da Silva J K L 2004 Phys. Rev. Lett. 93014101

[24] Leonel E D and McClintock P V E 2005 J. Phys. A: Math. Gen. 38823

[25] Saitô N, Hirooka H, Ford J, Vivaldi F and Walker G H 1982 Physica D 5273

[26] Bohigas O, Boosé D, Egydio de Carvalho R and Marvulle V 1993 Nucl. Phys. A 560197 